\title{
Uma abordagem para adequação de serviços baseada em práticas da ITIL
}

\author{
An approach to service suitability based on ITIL practices \\ Luiz Oscar Topin, Eduardo N. Borges, Luciano Maciel Ribeiro \\ Centro de Ciências Computacionais \\ Universidade Federal do Rio Grande \\ Av. Itália, km 8, 96203-900, Rio Grande - RS \\ Itopin@gmail.com, \{lucianoribeiro,eduardoborges\}@furg.br
}

\begin{abstract}
RESUMO
Neste artigo são identificadas fragilidades na governança de TI em empresas de todo o Brasil que possuem os seus principais processos de negócio apoiados no setor de TI, ou que são provedores diretos de serviços de TI. Itens específicos foram levados em conta a fim de medir o nível de governança dessa empresas. Com base nas melhores práticas da ITIL V3, foram propostas soluções para contemplar e solucionar as fragilidades encontradas através desta pesquisa. Estas soluções foram testadas em duas empresas distintas: uma fábrica de software e uma empresa do setor de óleo e gás em que a TI é essencial para seu modelo de negocio. Em contato direto com os gestores destas empresas, os artefatos para adequação de serviços de TI foram aperfeiçoados e generalizados.
\end{abstract}

\section{Palavras-Chave}

Governança de TI, gerenciamento de serviços, ITIL.

\begin{abstract}
This paper identifies weaknesses in IT governance in companies all over Brazil that have their main business processes supported in the IT sector, or that are direct providers of IT services. Specific items were taken into account in order to measure the level of governance of these companies. Based on ITIL V3 best practices, solutions were proposed to contemplate and solve the fragilities found in this research. These solutions have been tested in two distinct companies: a software factory and an oil and gas company in which IT is essential to their business model. In direct contact with the managers of these companies, the artifacts for adequacy of IT services have been perfected and generalized.
\end{abstract}

Permission to make digital or hard copies of all or part of this work for personal or classroom use is granted without fee provided that copies are not made or distributed for profit or commercial advantage and that copies bear this notice and the full citation on the first page. To copy otherwise, or republish, to post on servers or to redistribute to lists, requires prior specific permission and/or a fee.

SBSI 2017 June $5^{\text {th }}-8^{t h}$, 2017, Lavras, Minas Gerais, Brazil

Copyright SBC 2017.

\section{CCS Concepts}

-Applied computing $\rightarrow$ IT governance; Business process management;

\section{Keywords}

IT governance, service management, ITI

\section{INTRODUÇÃO}

Há muito tempo o cenário corporativo está passando por uma transformação. O setor de Tecnologia da Informação (TI), anteriormente considerado apenas um setor de suporte operacional, vem obtendo cada vez mais importância. Hoje este setor faz parte do alinhamento estratégico e do núcleo de negócios das empresas, tornando-se essencial para a tomada de decisão.

Ter uma utilização plena e eficaz da TI é preciso primeiro controlar para poder gerenciar a TI, e então torna-la veículo para agregar valores aos produtos e serviços da organização. Essa é uma das principais preocupações da Gestão de TI que visa colocar a TI como fator estratégico aos negócios da organização.[3]

Para atingir o sucesso no uso da TI é necessário administrar uma série de recurso e serviços. Esta tarefa exige um grande esforço por se tratar de serviços com alta complexidade, porém essenciais. Sem um gerenciamento eficiente, estes serviços poderiam prejudicar o andamento de projetos principalmente devido a falta de planejamento, necessidade de alinhamento com o negócio ou ainda outro fator crítico para a manutenção dos serviços de TI da corporação. Portanto, como utilizar esses recursos da melhor maneira possível, garantindo que os investimentos feitos em TI terão o retorno desejado e principalmente, como utilizar a TI como uma estratégia competitiva dentro da corporação?

Diversas metodologias, manuais e frameworks foram desenvolvidos e testados com a finalidade de buscar uma melhor solução para os problemas de infraestrutura de TI. Neste artigo serão abordadas boas práticas da Information Technology Infraestructure Library (ITIL), considerada por muitos autores como o mais consagrado conjunto de práticas para gerenciamento de serviços de TI. O objetivo deste trabalho é propor soluções baseadas na ITIL que minimizem as fragilidades de empresas que não possuam governança de TI ou que seus processos de negócio não estejam alinhados ao setor de TI. Foram desenvolvidos uma série de artefatos a partir de dados públicos disponibilizados pelo Tribunal de 
Contas da União (TCU). Os resultados deste trabalho foram testados em duas empresas de diferentes setores com a finalidade de verificar a viabilidade da implantação das soluções propostas e o impacto nos processos de negócio.

O restante do texto está organizado da seguinte maneira. Na Seção 2 são introduzidos conceitos essenciais para o entendimento do trabalho, incluindo o detalhamento sobre os componentes da ITIL. Na Seção 3 é apresentada a metodologia de pesquisa utilizada. Os resultados alcançados e dois estudos de caso são detalhados na Seção 4. Por fim, a Seção 5 apresenta as considerações finais e discute alguns trabalhos futuros.

\section{FUNDAMENTAÇÃO TEÓRICA}

O termo governança de TI tem suas origens no conceito de governança corporativa. Ela pode ser definida como o conjunto estruturado de políticas, normas, métodos e procedimentos destinados a permitir à alta administração e aos executivos o planejamento, a direção e o controle da utilização atual e futura da Tecnologia da Informação [2]. A governança de TI deve assegurar, a um nível aceitável de risco, a eficiente utilização de recursos, apoio aos processos da organização e o alinhamento estratégico. Seu principal objetivo é garantir que o uso da TI agregue valor ao negócio da organização.

A Governança de TI busca o compartilhamento de decisões de TI com os dirigentes da organização, assim como estabelece as regras e os processos que nortearão o uso da TI pelos usuários, departamentos, divisões, fornecedores e clientes, determinando como a TI deve prover os serviços para a empresa [4].

A gestão de TI busca governar a área de TI de forma que agregue valor aos negócios da organização. [3] Afirma que organizações com uma governança de TI ajustada ao negócio, com foco no tratamento adequado da informação, têm suas ações facilitadas no intuito de aproveitar as oportunidades e correm menos riscos diante das ameaças potenciais.

Um processo pode ser definido como um conjunto de atividades coordenadas combinando e implantando recursos e habilidades com o objetivo de produzir uma saída, a qual cria valor para um cliente ou parte interessada. Cada processo deve ter o máximo de detalhamento possível e descrever o que cada atividade deve executar. Os procedimentos de um processo não devem necessariamente iniciar e ser finalizados dentro do mesmo departamento, podendo passar por dois ou mais durante o seu ciclo de vida.

Já os serviços de TI são uma combinação de hardware, software, processos e pessoas. Um serviço é conjunto de TI e não-TI, mantidos por um provedor de TI, cujo objetivo é satisfazer uma ou mais necessidades de um cliente (áreas de negócio) e suportar os objetivos estratégicos do negócio do cliente [5]. Eles ainda podem ser definidos como um meio de entregar valor aos clientes facilitando resultados sem arcar com os riscos e custos específicos. Há uma variedade de contextos em que a definição de serviço pode ser aplicada, mas o conceito básico, é de que um serviço é o meio de entrega e valor [7].

\subsection{ITIL V3}

A ITIL oferece uma abordagem sistemática para a entrega de qualidade dos serviços de TI. Ele dá uma descrição detalhada da maioria dos processos importantes em uma organização de TI, e inclui listas de verificação para tarefas, procedimentos e responsabilidades que podem ser utilizados como uma base para a adaptação às necessidades de organizações individuais [1].

A ITIL foi desenvolvida no final dos anos 80 pelo governo britânico, primeiramente pela Central Computer and Telecommunications Agency (CCTA) e posteriormente pela Office of Government Commerce (OGC), a partir da necessidade do governo de ter seus processos organizados na área de TI. O resultado foi a junção dos melhores processos e práticas para ancorar a gestão dos serviços de TI.

Em sua primeira versão a ITIL consistia em aproximadamente 40 livros, como um esforço para: padronizar, monitorar, metrificar e garantir a qualidade dos serviços de TI contratados pelos diferentes órgãos, agências e instituições governamentais da Inglaterra, melhorando a eficiência de seus investimentos e entrega de seus projetos de TI. Entre 1990 e 2004 foi feita uma revisão na ITIL. A versão 2 se tornou mais estruturada e melhor direcionada, com apenas 9 livros, criando uma ponte entre a tecnologia e os negócios, fortemente focado nos processos necessários para prestar serviços eficazes para os clientes dos negócios. A terceira edição denominada ITIL V3, lançada em 2007, organiza os processos de gerenciamento de serviços em apenas 5 livros.

O principal objetivo da ITIL é prover um conjunto de práticas de gerenciamento de serviços de TI testadas e comprovadas no mercado (organizada segundo uma lógica de ciclo de vida de serviços), que podem servir como balizadoras, tanto para organizações que possuem operações de TI em andamento e pretendem empreender melhorias, quanto para criações de novas operações [4]. A ITIL descreve a base para a organização dos processos da área de TI, visando sua orientação para o gerenciamento de serviços de TI. As diversas práticas reunidas descrevem os objetivos, atividades gerais, pré-requisitos necessários e resultados esperados dos vários processos, os quais podem ser incorporados dentro das áreas de TI [5].

Embora a ITIL defina um conjunto de boas práticas para gestão de TI, ela não é uma metodologia. A biblioteca é composta por um senso comum na abordagem do gerenciamento de serviços, com a finalidade de entregar valor ao negócio.

A Figura 1 apresenta a abordagem de ciclo de vida, que permite que se tenha uma visão do gerenciamento de serviços pela perspectiva do próprio serviço, em vez de focar em cada processo ou prática por vez. Essa característica realça mais um importante objetivo, que é mensurar e gerenciar o valor que os serviços de TI efetivamente adicionam ao negócio [4]. Os livros em destaque nesta figura são:

- Estratégia de Serviço (Service Strategy);

- Projeto de Serviço (Service Design);

- Transição de Serviço (Service Transition)

- Operação de Serviço (Service Operation);

- Melhoria Contínua de Serviço (Continual Service Improvement). 


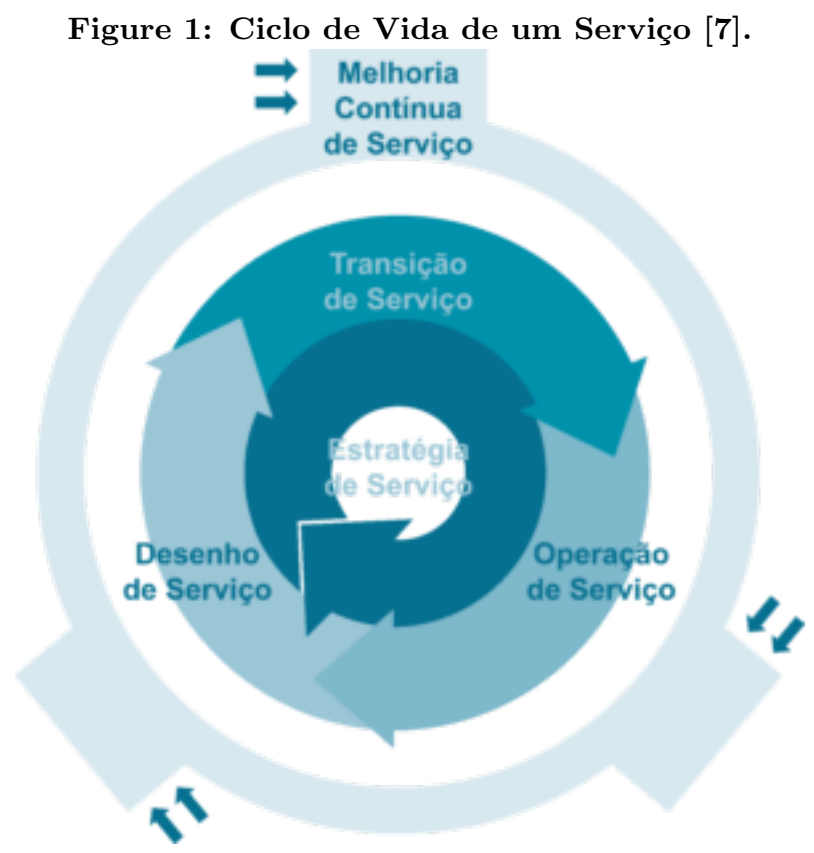

\subsection{Estratégia de serviço}

A Estratégia de Serviço é a primeira fase do ciclo de vida e é o eixo central que move as outras fases. É durante esta fase que as políticas e os objetivos de um serviço são definidos como, por exemplo, o valor que este serviço irá entregar ao negócio. Dentro da fase de Estratégia de Serviço existem 3 principais processos, que são: gerenciamento do portfólio de serviços, gerenciamento de demanda e gerenciamento financeiro [6].

Esta fase pode ser considerada um guia para definir os objetivos e as expectativas de desempenho em relação aos serviços prestados aos clientes e espaços de mercado, e também para identificar, selecionar e priorizar oportunidades. Ela tem como objetivo garantir que as organizações estão em posição de lidar com os custos e riscos associados com a seu portfólio de serviços, e que são criados não apenas para uma eficácia operacional, mas para um desempenho diferenciado [7].

\subsubsection{Gerenciamento de portfólio de serviços}

O processo de gerenciamento de portfólio de serviço é responsável por gerenciar o portfólio de serviços durante todo o ciclo de vida de um serviço, focando no valor que os serviços entregam ao negócio [6]. O gerenciamento de portfólio descreve os serviços de um provedor de serviços em termos de valor de negócio, formulando a necessidade de negócios e a reação do prestador de serviços.

O portfólio está dividido em três partes:

- o funil ou pipeline de serviços, que mostra o que está na fila para ser desenvolvido;

- o catálogo de serviços, que mostra os serviços que estão atualmente em operação;

- os serviços obsoletos, que mostra os serviços que devem ser aposentados ou que estão fora de operação.

Na prática, o portfólio de serviço é um repositório do qual serão obtidas as informações sobre os serviços e sua situação em todo o ciclo de vida. O uso eficiente do portfólio contribui para o retorno sobre o investimento e na redução dos riscos de um serviço [6].

\subsection{Transição de serviço}

O grande propósito da fase de transição de serviço é garantir que serviços novos, modificados ou descontinuados atendam às expectativas do negócio conforme documentadas nos estágios anteriores. Esta fase é composta pelo seguinte conjunto de processos e atividades para a transição do serviço no ambiente de produção:

- gerenciamento de mudanças;

- gerenciamento de configuração;

- gerenciamento de liberações.

A fase de transição de serviço abrange a transição de um serviço para o ambiente de operação, seja novo ou modificado, garantindo com isto que os requisitos da estratégia incluídos no desenho sejam realizados na operação, controlando os riscos de falhas e interrupções [6].

\subsubsection{Gerenciamento de mudanças}

$\mathrm{O}$ processo de gerenciamento de mudanças visa administrar o processo de mudança, buscando limitar o surgimento de erros relacionados à sua implementação. Através desse processo, todas as implementações e atualizações na infraestrutura de TI serão analisadas e planejadas, para que sua execução ofereça menor risco e impacto para o negócio. [6]

A mudança é a passagem de um estado para outro. Portanto, o gerenciamento de mudanças é importante, pois garante a existência de métodos e procedimentos para o atendimento rápido e eficiente em todas as situações de mudanças. Ela também garante que as mudanças em itens de configuração sejam registradas na respectiva base de dados [5].

Algumas das atividades consideradas no gerenciamento de mudanças são:

- Planejamento e controle das mudanças;

- Programação das mudanças e das implantações;

- Comunicação entre os envolvidos;

- Autorização das mudanças;

- Criação de planos de remediação;

- Relatórios;

- Definição do impacto da mudança;

- Melhoria contínua.

O gerenciamento de mudanças é responsável pela gestão de mudanças que envolvam hardware, equipamentos e softwares de comunicação ou ainda sistemas e aplicações. O objetivo do gerenciamento de mudanças é garantir que as mudanças sejam registradas, planejadas, aprovadas e implementadas por meio do uso de métodos e procedimentos padronizados e que o gerenciamento de mudanças decide e coordena. 


\subsubsection{Gerenciamento de liberação}

A ITIL define o objetivo do processo de gerenciamento de liberação como sendo a construção, testes e fornecimento dos serviços especificados no projeto de serviços, atendendo assim aos requisitos e objetivos das partes interessadas. $\mathrm{O}$ processo de gerenciamento de liberação faz a administração, além da distribuição das versões de software e hardware utilizadas no ambiente de produção. Essas versões recebem suporte do departamento de TI, garantindo assim o nível de serviço exigido [6].

Fazem parte do processo de gerenciamento de liberação:

- política de liberação e planejamento;

- projeto, construção e configuração da liberação;

- teste e aceite da liberação;

- planejamento da implementação;

- comunicação, preparação e treinamento;

- distribuição e instalação da liberação.

\subsection{Operação de Serviço}

O principal objetivo da operação de serviços é coordenar e realizar as atividades e processos necessários para entregar e gerenciar serviços, de acordo com os níveis de serviços acordados com os clientes e usuários dos sistemas. A operação de serviços também é responsável pelo gerenciamento contínuo da tecnologia utilizada para fornecer e dar suporte aos serviços prestados.

Os processos bem concebidos e bem implementados terão pouco valor se a operação diária desses processos não forem devidamente conduzidos, controlados e geridos. É na fase de operação de serviço que o valor do serviço é percebido pelos clientes. Nesta fase são definidos os processos:

- gerenciamento de incidentes;

- gerenciamento de eventos;

- cumprimento de requisição;

- gerenciamento de problemas;

- gerenciamento de acesso.

A Operação de Serviço conduz, controla e gerencia as operações do dia-a-dia, monitorando o desempenho, levantando métricas e informações de forma sistemática, para habilitar a melhoria de serviço continuada [7].

Os principais objetivos da operação de serviços são coordenar e executar as atividades e processos necessários para entregar e gerenciar os serviços nos níveis acordados; conduzir, controlar e gerenciar as operações do dia a dia; gerenciar a tecnologia utilizada para a entrega e suporte aos serviços e; monitorar o desempenho, levantar métricas e informações de maneira sistemática para se habilitar a melhoria continuada de serviço [6].

\subsubsection{Gerenciamento de incidentes}

O principal objetivo do gerenciamento de incidentes é garantir que, após a ocorrência de um incidente, um serviço seja restabelecido o mais rápido possível ao seu estado normal, minimizando os impactos que este incidente pode causar ao negócio. Um incidente é qualquer evento que interfere no funcionamento normal de um serviço de TI, causando, ou podendo causar, sua interrupção, a qual prejudica sua qualidade [7].

O processo de gerenciamento de incidentes se inicia a partir de incidentes apontados pelos usuários ou por ferramentas de monitoramento. Em um primeiro momento, a resolução de um incidente é o foco principal, pois o serviço precisa ser restabelecido o mais rápido possível. Isto, muitas vezes, pode ser alcançado através da aplicação de uma medida de contorno [5].

Os objetivos do processo de gerenciamento de incidentes são:

- solucionar o incidente de maneira rápida, cumprindo os acordos de nível de serviço estabelecidos;

- diminuir os impactos que o incidente pode causar ao negócio;

- manter a comunicação aos usuários sobre o estado dos incidentes;

- avaliar os incidentes e suas possíveis causas, informando ao processo de gerenciamento de problemas;

- assegurar os melhores níveis de disponibilidade e desempenho aos serviços de TI.

O escopo do processo de gerenciamento de incidentes abrange desde o tratamento de qualquer evento que interrompa ou possa interromper o funcionamento de um serviço de TI até reporte pelo usuário, ferramentas de monitoramento ou pela própria equipe técnica. As atividades do processo de gerenciamento de incidentes são:

- detecção e registro do incidente ? o incidente é detectado ou comunicado, e cria-se para ele um registro;

- classificação e suporte inicial ? o incidente é classificado por tipo, impacto, urgência e prioridade. Caso se trate de uma solicitação de serviços, aplica-se o procedimento específico previsto;

- diagnóstico e investigação ? se não existe uma solução conhecida, o incidente será investigado;

- resolução e recuperação ? assim que a solução de contorno ou definitiva for encontrada, deve ser aplicada. Se uma mudança for necessária, dispara-se uma requisição de mudança, que será tratada pelo processo de gerenciamento de mudanças;

- encerramento ? verifica-se se o usuário está satisfeito com a resolução e fecha-se o incidente. Esta etapa inclui a atualização dos detalhes de resolução do incidente e a comunicação com o usuário.

\subsubsection{Gerenciamento de Problemas}

Um problema define uma situação indesejável, de causa raiz desconhecida, e que se refere a um ou vários incidentes ocorridos [7]. O principal objetivo do gerenciamento de problemas é encontrar a causa raiz dos incidentes e impedir que voltem a acontecer.

O gerenciamento de problemas inclui atividades preventivas e reativas. No aspecto reativo busca-se a resolução dos problemas em resposta aos incidentes ocorridos. Já o aspecto preventivo visa impedir a recorrência dos incidentes, 
identificando fragilidades na infraestrutura de TI, fazendo propostas para eliminá-las, antes que ocorram novos incidentes [6].

Para o trabalho descrito neste artigo, o processo de gerenciamento de incidentes e o processo de gerenciamento de problemas, por serem bastante semelhantes, foram tratados no mesmo documento, buscando uma maior agilidade e facilidade no preenchimento correto do documento.

\section{METODOLOGIA DE PESQUISA}

A metodologia deste trabalho foi dividida em três estágios. O primeiro estágio consiste na definição dos dados secundários a serem utilizados. É efetuada uma categorização e reorganização dos mesmos, aplicando um processo de estatística descritiva. Após os dados são analisados e é verificada a abrangência dos processos da ITIL cobertos pelas empresas estudadas.

O segundo estágio propõe um conjunto de artefatos cuja finalidade é habilitar as empresas a realizarem todos os processos de governança de TI passíveis de implementação pelas empresas em questão. É realizada uma rodada de reunião com os gestores das empresas selecionadas. Utilizando como parâmetro as melhores praticas da ITIL V3, uma série de documentos padronizados foram são propostos para os gestores verificarem se os processos desenhados no documento poderiam se adaptar aos processos de negócio das suas empresas. Sugestões destes gestores são levadas em conta com a finalidade de adaptar os documentos a fim de contemplar os principais processos internos das empresas, sem atrapalhar ou comprometer os atuais fluxos de negócios.

No terceiro estágio os artefatos são aplicados nas empresas para verificar a viabilidade inicial das novas dinâmicas de trabalho e o impacto gerado no fluxo de trabalho e processos de negócio. Uma nova rodada de reuniões é realizada com os gestores das empresas e fim de recuperar o feedback sobre a aplicação dos artefatos e novas sugestões de adaptações a serem feitas.

Os últimos dois estágios podem ser repetidos até que a solução se adeque à realidade das empresas.

\section{RESULTADOS}

No ano de 2014, o Tribunal de Contas da União (TCU) realizou uma pesquisa em 372 empresas públicas que utilizam TI, a fim de avaliar o nível de Governança Corporativa e Governança de TI. Os dados foram coletados pelo TCU e disponibilizados em planilha eletrônica onde, de forma pública e gratuita, qualquer indivíduo pudesse ter acesso aos dados. foram selecionados os dados contidos na base de dados em formato CSV disponível eletronicamente ${ }^{1}$. Um novo ciclo de coleta iniciou em 2016, mas os dados ainda não estavam disponíveis na época da escrita deste artigo.

Das 147 perguntas contidas no questionário, apenas as 7 perguntas relativas a ITIL v.3 e referentes a categoria de processos foram consideradas neste trabalho. O questionário disponível para consulta na Web aceita respostas utilizando números de 1 a 5 , de acordo com os critérios estabelecidos no próprio questionário, em que 2 seria não adota e 5 adota integralmente.

Empresas que responderam as opções não adota e adota integralmente foram contabilizadas. As opções adota parcialmente, iniciou plano para adotar e não se aplica foram

${ }^{1}$ https://goo.gl/Q97Plh descartadas da análise dos dados. Este critério foi adotado por não ser possível mensurar o nível de governança utilizado pelas empresas que marcaram as opções acima.

Após filtrar os dados coletados, utilizando os critérios de escolha já estipulados anteriormente, os resultados obtidos estão descritos na Tabela 1, que apresenta o número de empresas que adota ou não cada processo da ITIL analisado.

Utilizando como base as respostas dadas pelas empresas estudadas, foram desenvolvidos seis documentos contendo um padrão para processos e serviços, contendo planos e estratégias cuja finalidade é alinhar a TI dos negócios, padronizar o fluxo de trabalho, gerar valor para o negócio e otimizar as estratégias de negócio. Os documentos criados foram:

- Documento de gerenciamento de mudanças e liberação;

- Documento de gerenciamento de portfólio de serviços;

- Documento de gerenciamento de incidentes e problemas;

- Documento de gerenciamento de implantação;

- Documento de gerenciamento de continuidade.

Cada artefato possui a sua finalidade e podem ser usados individualmente, de acordo com a necessidade da corporação. Todos os artefatos desenvolvidos estão disponíveis de forma pública na $\mathrm{Web}^{2}$ em um único arquivo compactado de formato RAR e não estão no corpo do artigo por restrições de espaço evidentes no limite de páginas permitido.

O documento de gerenciamento de mudanças e liberação tem como objetivo desenhar o processo de gerenciamento de mudanças e liberação, com a finalidade de definir as políticas de liberação e mudanças, o controle de versão dos recursos existentes, controlar a distribuição de versões confiáveis, mudanças emergenciais e frequência de atualização. Assim, este artefato garante que todas as implementações e alterações na infraestrutura de TI serão analisadas e planejadas para que se tenha o menor risco e impacto no ambiente de produção e durante a fase de implantação.

O documento de gerenciamento de portfólio de serviços tem como objetivo identificar a lista completa de serviços oferecidos e gerenciados pelo provedor de serviços, onde a intenção é documentá-los. Neste artefato são acordadas as metas de serviços, os detalhes dos usuários e dos responsáveis pela manutenção contínua de cada serviço.

O objetivo do documento de gerenciamento de incidentes e problemas é de reestabelecer o serviço o mais rápido possível e minimizar o impacto negativo no negócio, garantindo os melhores níveis de disponibilidade e qualidade dos serviços oferecidos. Espera-se que estes serviços sejam mantidos conforme os acordos de nível estabelecidos. O gerenciamento de incidentes e o gerenciamento de problemas estão juntos no mesmo documento devido ao relacionamento forte entre os processos, pois os problemas são causas de um ou mais incidentes.

O documento de gerenciamento de implantação é composto por duas seções, a Estratégia de Implantação e o Plano de Implantação. A primeira é usada para formular uma abordagem de implantação para o aplicativo ou sistema e é concluída no início do projeto. A segunda seção contém

${ }^{2}$ https://goo.gl/QZBpJG 
Table 1: Quantidade de empresas que adotam ou não processos da ITIL.

\begin{tabular}{lrr}
\hline Processo ITIL & Adotam & Não adotam \\
\hline Gerenciamento do catálogo de serviços & 34 & 103 \\
Gerenciamento de continuidade dos serviços & 25 & 121 \\
Gerenciamento de mudanças & 41 & 139 \\
Gerenciamento de configuração e ativos & 30 & 110 \\
Gerenciamento de liberação e implantação & 35 & 147 \\
Gerenciamento de incidentes & 100 & 84 \\
Gerenciamento de problemas & 45 & 121 \\
\hline
\end{tabular}

informações detalhadas de cronograma, recursos, técnicas e suporte necessárias para a implementação bem sucedida.

Por fim, o documento de gerenciamento de continuidade tem como objetivo o planejamento de estratégias e procedimentos com a finalidade de minimizar o impacto da indisponibilidade de serviços nos processos de negócios dos clientes. A intenção é antecipar e minimizar o impacto da indisponibilidade de sistemas, através do desenvolvimento de procedimentos pré definidos e testados, levando em conta dados, software, hardware e aplicações chave.

Para a validação dos artefatos propostos, duas empresas foram escolhidas, a Refinaria de Petróleo Riograndense SA, que produz e comercializa produtos derivados de petróleo, e a Compasso, empresa do ramo de desenvolvimento de software. Os gestores das empresas estudadas preencheram os documentos propostos, com dados referentes aos seus processos de negócio, a fim de garantir que as soluções desenvolvidas poderão se encaixar no seu fluxo de trabalho.

As ferramentas foram validadas em múltiplas etapas. Em um primeiro momento os gestores analisaram os documentos e verificaram a viabilidade de implantação nos seus fluxos de trabalho. Sugestões foram feitas pelos gestores e adaptações nos modelos foram realizadas, para que fosse possível encaixar efetivamente os modelos nas empresas escolhidas. Os processos que já possuíam um fluxo determinado foram escolhidos a fim de comparar a efetividade dos artefatos desenvolvidos, ou seja, se os documentos atendiam a todos os requisitos dos fluxos de trabalho que já eram executados dentro das empresas estudadas. Então uma nova rodada de avaliação, sugestões e adaptações foram executadas, até os documentos se encaixarem em definitivo, de forma eficaz e eficiente em todos os processos e serviços.

\section{CONCLUSÃO}

É possível perceber que as práticas propostas pela ITIL V3 podem ser aplicadas em uma variedade de empresas, de grande e médio porte, que possuem os seus processos de negócio puramente baseados em TI ou em setores que utilizam TI apenas para manter os processos da corporação. É necessário que estas empresas possuam os seus processos bem definidos e um fluxo de trabalho já estabelecido. Uma equipe motivada e disposta a implantar mudanças também é fundamental, visto que as práticas da ITIL devem ser adaptadas em diferentes estruturas organizacionais.

Não apenas as equipes devem ser preparadas para implantação das práticas da ITIL, mas toda a empresa deve ser preparada. Os gestores devem estar motivados e cientes das mudanças que ocorrem quando uma nova prática é implantada no ambiente de trabalho, e que estas mudanças devem afetar todo o fluxo de trabalho. Além disso, adaptações devem ocorrer, não só nas práticas, mas em todo o ambiente organizacional.

Processos de negócios funcionam melhor e agregam mais valor quando possuem uma estrutura organizada e um fluxo de trabalho padronizado, onde cada envolvido pode conhecer detalhes do andamento de cada serviço sem a necessidade de uma explicação direta. As melhores práticas da ITIL possuem uma vasta e detalhada coleção de práticas utilizadas na padronização de processos, fazendo dela uma ferramenta essencial em empresas que possuem um alto grau de complexidade nos seus processos de negócio.

Apesar das melhores práticas serem muito detalhadas, específicas e possuírem uma complexidade relativamente alta, a ITIL V3 é bastante maleável e adaptável, podendo ser implantada em uma série de ambientes organizacionais. É necessário considerar quais setores da organização irão receber ou estão preparados para receber as práticas da ITIL, e quais fases do ciclo de vida serão aplicados. É preciso estudar a organização e identificar as melhores aplicações entre as melhores práticas, para não engessar os processos de negócio da corporação.

Contudo, é possível perceber que os artefatos propostos atendem aos critérios estabelecidos. Eles podem ser preenchidos rapidamente e sem dificuldades, visto que cada campo possui o seu detalhamento. Se necessário, os artefatos podem ser adaptados e ajustados conforme as necessidades ou os critérios estabelecidos pelas empresas. Finalmente, os documentos podem ser entendidos por indivíduos que estejam ligados direta ou indiretamente com os processos.

\section{REFERENCES}

[1] J. V. Bon. Foundations of IT Service Management Based on ITIL V3. Van Haren Publishing, ZaltBommel, 2007.

[2] T. C. U. Brasil. Acórdão 16 2.308/2010, plenário, diário oficial da união, brasília, 2010.

[3] D. de Mello Benzi, J. S. Neto, T. R. C. Orlandi, and R. de Souza Jr. Governança de tecnologia da informação: facilitadora na estratégia organizacional. In Simpósio de Excelência em Gestão e Tecnologia, 2006.

[4] A. Fernandes and F. Abreu. Implantando a Governança de TI - da Estratégia À Gestão Dos Processos e Serviços. Brasport, 2008.

[5] I. Magalhães and W. Pinheiro. Gerenciamento de Serviços de TI na Prática. Novatec, 2007.

[6] A. Mülbert, M. Júnior, and G. Santana. Gerenciamento de Serviços de TI pelo Modelo ITIL. Unisul Virtual, 2011.

[7] OGC, United Kingdon. The Official Introduction to the ITIL Service Lifecycle, Janeiro 2007. 